structure, and no tubercles were found in them, but the right lung was conpressed. A copious deposit of tubercle was found in the bronchial glands. The heart was healthy. The liver was stulded with masses of crude tubercle, and the glands in its neighbourhood were also affected.

REMaris. The above cases are quoted as somewhat rare examples of the very common diseases which they illustrate. In the former, hardly any of the symptoms of malignant disease, either of the lungs or kidneys, were present; nor, although it was evident that the man was affected with some mortal dis. ease, was it possible to form a diagnosis in the absence of any prominent symptom. The epileptic convulsion with which he was once seized was evidently only a symptom, as it never recurred, and was clearly referrible to the poisoned blood which was circulating in the body. It was to the latter cause, no doubt, more than to the local ailments, that death was to be ascribed; and the case may be regarded as an instance of malignant disease rapidly developed, and proving fatal before its presence had set up sufficient functional disturbance to give rise to distinet symptoms.

The other case was almost equally obscure; and here also the probalility is that the patient's death was induced by the discase of the blood, inasmuch as the local ailment had not remarkably increased cluring the latter period of his life. The increase of dyspuma, and the consequent rapid sinking, was perhaps due to some suddenly increased pressure upon the lung by the abscesses, or to some sudden deposit under the rius; but the pressure was not so great as to have proved fatal to a man whose cireulating lluid was in a healthy condition.

\section{Orininal Communtations.}

\section{ON DISEASES OF JOINTS.}

By Homms Cootr, Esq., F.R.S.C., Assistant-Surgeon to St. Bartholomew's Hospital, and to the Royal Orthopædic Hospital, etc.

\section{TI II A NKLE-JOINT. \\ [Continued from page 307 .]}

T.unIres varus, or club.foot, consists in the elevation of the hecl from contraction of the muscles of the calf; inversion of the font, with semirotation of its anterior part by the contrac. tion of the anteriur and posterior tibial muscles; and shorten ing of the sole by contraction of the plantar fascia, muscles, ligaments, etc. In young children, in whom the feet have not yet sustained the weight of the body, the deformity is less than in adults, and there is no remarkalle abnormity in the articular surfaces and their uniting structures.

I dissected the feet of a still-born infant, which came into the world with double talipes varus. If the altered direction of the foot, and the shortness of the tendons, be excepted, there was no unnatural appearance to be recorded. I have at the present time under my care an infant in whom there is a slight inversion of the right foot, the chief deformity being increas in the transverse arch. Here mechanical treatment alone will suffice to effect a cure.

In the more advanced stages of talines varus the os calcis the astragalus, the os scaphoides, and the os cuboides undergo remarkable changes, both in form and direction; new articula tions are formed; the normal articular surfaces retain only in part their proper relations; those parts which are exposed either lose their cartilaginous covering, or are coated with a fibrous, or fibro-cartilaginous tissue.

Many years ago I dissected in a subject, aged 60 , the feet which were both affected with talipes varus. The man had walked for many years by the aid of crutches, the dorsum of the feet resting on the ground, the sole being turned upwards and inwards. Under the dorsal integuments, which were very much thickened, there was a large and strong-walled bursa, subdivided by fibrous bands; there was very great increase in the thickness of all the tendonous theca. The tendons of the extensor communis dicitorum and peroneus tertius were united ly strong short fibrous bands; the tendon of the latter muscle was very thick and strong. 'The tendon of the extensor proprius pollicis was tightly bound down in its sheath. The sole of the foot was turned upwards and inwards by the action of the following contracted muscles : tibialis anticus, and posticus; ex- tensor proprius pollicis, abductor pollicis, flexor communis digi torum. The plantar fascia was very tense. The body rested upon the astragalus, os cuboides, onter and middle cuneiform bones, and in some degree the metatarsus and the tarsal extremity of the os calcis. The direction of the os calcis, from contraction of the muscles of the calf, inclined to be vertical. After division of all the tendons, the interosseous ligamentous bands prevented the feet from returning to their normal position. 'T'he bones of the lower extremity, of normal length, were light and greasy.

Dittel found, in a similar case of talipes varus, all the sof parts on the convexity of the foot stretched, elongated, relaxed, and imperfectly nourished; some muscular slips in a state of fatty degeneration. On the concavity the soft parts were shortened, tense, and better nourished. The bone, which took direct share in the deformity were the os calcis, the astragalus, and the tibia; the ligaments which were implicated were those on the inner border of the foot, especially the deltoil ligament (internal lateral) and the calcaneo-scaphoid.

The parts requiring subcutaneous section in this deformity are the tendons of the anterior and posterior tibial muscles, and the tendo Achillis. For the mode of performing these operations, and for the order of succession, I must refer to works on orthopædic surgery. I would here merely remark, that in practice we meet with some curious instances of the use of the tenotomy-knife. A woman is at present under my care for the treatment of non-congenital talipes varus of the left foot. She had undergone an operation a few years beforc coming to the Orthopædic Hospital, and had had divided the tendo Achillis, the plantar fascia, and the flexor longus pollicis. The only result of this proceeding was a painful affection in the ball of the great toe, the deformity of the foot remaining unchanged. In another case the surgeon occupied himself in dividing the tense plantar fascia. Experience shows that this structure (the contractions of which are always secondary) yields readily to moderate extending force. Division of its fibres is always attended with the inconveniencies of a new cicatrix.

The only part of the operation attended with difficulty and some amount of risk is the division of the tendon of the tibialis posticus muscle; and I am perfectly certain that in very many instances it is never divided at all. The tendon lies so close to the bone, that any obliquity in the direction of the knife sends the point away from the part to be divided. In infants this accident happens not uncommonly, and the operation is reported to have failed, the truth being that the most important part of the proceeding has miscarried; for until the tendon of the tibialis posticus muscle is divided, the treatment is usually unsatisfactory. The first person to propose and carry out the subcutaneous division of the tendon of the tibialis posticus muscle in infants in this country was Mr. Tamplin. About fifteen years ago he noticed the frequent failures in the usual method of treatment, and invented an instrument consisting of a knife lodged in a small thin and flat cannula. A puncture was first made into the sheath of the posterior tibial tendon; the knife and cannula were then introduced; and when the tendon was fairly caught, the knife was thrust out, so as to divide it without danger to the tibial artery. The late Mr. Bransby Cooner, who witnessed these operations, expressed a doubt as to the fact of the division of the tendon, until one day, when the small knife broke off in the limb of a patient upon whom Mr. Tamplin happened to be operating. 'There ensued an immediate necessity for enlarging the wound, when the broken piece was found in the situation where it should have lain, namely, between the tendon of the posterior tibial muscle and the bone.

The operation is now performed with a blunt pointed knife, the sheath of the tendon having been once opened. A wound of the posterior tibial artery is an accident which the surgeon would use every possible precaution to avoid, even in infants.

Twelve years ago, I witnessed an operation for club-foot performed by a surgeon upon a boy eleven years of age. $\mathrm{He}$ divided the tibialis anticus and posticus muscles, and the flexor communis digitorum. After three or four days, frequent hæmorrhage ensued from the puncture in the proximity of the posterior tibial artery. Compresses were applied, which produced a slough upon the inner ankle; and ultimately it was decided to cut down upon and to tie the artery. Considerable swelling came on. The tendons of the tibialis posticus, the flexor communis digitorum. and the flexor longus pollicis, sloughed. Suppurations formed in the sole of the foot, and upon the outer side, one of which burst near the external malleolus. The result was unsatisfactory as regards the deformity, 
solely in consequence of want of experience in the details of treatment.

We find that the situation of the posterior tibial artery and its relation to the adjacent tendons varies greatly. I have seen it sometimes a short distance from the tendon of the flex or communis digitorum, sometimes immediately behind or even under it; and, in performing subcutaneous operations, the surgeon should remember that, in club-foot, the groove on the tibia which holds the tendons is often so oblique that it would carry the point of a sharp knife directly into the artery. A modern author suggests that " the best way to avoid this vessel is to puncture the sheath of the tendon with a sharp tenotome introduced directly downwards, and then to divide it in a direction forwards away from the vessel." But I believe that, in the first part of the proceeding, there would be great risk of falling into the very danger it is wished to avoid; and, moreover, I doubt the possibility of dividing the tendon with any certainty by cutting in a direction from behind forwards, $i . e .$, towards the tibia. It is also recommended, by way of precaution, to divide the tendo Achillis first, so that the others may be rendered more tense before their section is undertaken but this advice should not be followed, because, first, in in fants, the tendo Achillis is required in its intecrity to steady the foot in the earlier stage of proceedings; and because, secondly, in the aduit, the contracted and deformed foot has to be unfolded and put into shape after the division of the anterior and posterior tibial tendon, before any operation is performed on the great tendon of the heel. The first step is to convert the pes varus into pes equinus, and then to rectify the latter deformity. The inward displacement of the tendo Achillis in club-foot, and its abnormal relation to the posterior tibial artery, are points too well known to need remark.

Duval (T'raité du Pied.bot, p. 16士) relates the particulars of a case of anchylosis of a rreat number of joints in a woman aged 35, the subject of talipes varus in both feet. "The joints between the greater number of tarsal and metatarsa bones were anchylosed. The ankle-joint (i.e., the tibioastragaloid) and the joints of the toes alone were movable: nevertheless, in the first, adduction only was possible; while abduction was prevented by a firm fibrous mass, which extended from the inner side of the tuberosity of the os calcis to the internal malleolus, the inner side of the neck of the astragalus, and, indeed, to the tuberosity of the os scaphoides. The tarsal bones were all changed in volume and form, and anchylosed one with another by osseous concretions and lamella Ossification was especially remarked between the three ossa cuneiformia and the os naviculare, which were locked together as in the sagittal suture; also the articulations of the three first metatarsal bones were anchylosed by osseous plates. The metatarsal bones were less voluminous than natural."

Now, as regards this case, I may say that Mr. Tamplin has not met with one similar during the course of his long experi ence; nor have I, in my pathological researches, seen such a specimen, unless, indeed, those cases be excepted in which acute rheumatic inflammation has attacked the tarsus, and led to rapid and extensive ulceration of the bones. In every case of deformity, as known by the name of pes varus, the bones retained their form, and the joints their molility, although the displacement was considerable and of long duration.

\section{[T'o be continued.]}

\section{REMARKS ON THE THEORY OF ELIMINATION IN THE TREATMENT OF DISEASE.}

By C. Handfield Jones, M.B., F.R.S., Physician to St. Mary's Hospital.

[Real before the Harveian Society.]

[Concluded fiom paye 331.]

Is syphilis, we have, I suppose, an undoubted instance of a poison being received into the circulation, and producing widespread morbid effects. Of the modus operandi of the two great reputed remedies, mercury and iodide of potassium, we do not know much. Our most certain information respecting the action of the first, points to it as a controller of sthenic inflammatory movement, and an absorber of effused fibrine. Whether it has really any peculiar specific action on the syphilitic poison, at least in arlults, is to me, as to many, a matter of some doubt. I see, certainly, that it makes to cease for a time the secondary local affections; but does it really get rid of their exciting cause? The following quotation from Mr. Erasmus Wilson's work justifies this doubt. After more than five pages devoted to the treatment of constitutional syphilis, he proceeds: "We may now suppose the first attack of constitutional fever, or secondary symptoms, to have passed away; but it does not therefore follow that the syphilitic poison is entirely banished from the bloorl; on the contrary, the probability is, that after the lapse of a few months, a second attack will occur, and after that we have a third, a fourth, and even more; the attacks at last becoming irregular, and putting on a new shape and new characters."

The author just quoted refers the beneficial effect of mercury to its acting as an eliminant, and approves of sweating and diluent drinks as adjuvant means; but I must think there are strong objections to this view. It is notorious that copious salivation (three or four pounds a day Boerhaave required) or bilious purging are anything but desirable in the mercurial treatment of syphilis; on the contrary, we are especially anxious to obtain the mildest possible perceptible effect, and we stop as quickly as we can any violent action. I incline strongly to think that, when mercury acts beneficially in constitutional syplilis, it is as a blood alterant, decomposing and destroying some abnormal albuminoid matter in the blood. The marked improvement which takes pince in the health of diseased children under its use seems quite independent of any noticeable elimination, and altogether consonant to the mode of action I have suggested. Beyond an empiric acquaintance with the action of iodide of potassium, we are quite in obscurity. We find that it effectually arrests certain inflammations of fibrous tissues, syphilitic or rheumatic, notably those of the periosteum; that it is prone to cause irritation of some mucous surfaces; and that it exerts an alssorbent action, attended with more or less wasting, if long continued. Dr. Pereira states that diuresis is a common consequence of its use. This I have not observed. I took ten grains in five doses, in about thirty hours, and collected and analysed the whole urine before and after. Five days before taking the iodide, the twenty-four hours' amount was 32 ounces, of syecific gravity $1028 \cdot 5$. While I was taking the iodide, the amount was $38^{\circ} 5$ ounces, of specific gravity 1(120): the urine contained iodine. The urea, the uric acid, the acidity, the chlorine, phosphoric and sulphuric acid, were all diminished by the use of the drug; the urea especially was less by 170 grains. In the case of a man with incomplete paralysis, which I suspected might be due to the poison of leal, and who had been taking 30 grains a day for several days, the twenty-four hours' amount of urine, while under the influence of the iodide, was 50 ounces, of specific gravity 102:3; it contained iodine. Six days after omitting the iodide, and having substituted for it strychnia and arnica, the twenty-four hours' amount was 62 ounces, of specific gravity 1013. The urea had increased, and the sulphuric acid; the phosphoric acid had diminished, and the uric acid was 0 . The result of these experiments ithough far too few to be decisive) scarcely favours the view that iodide of potassium causes increased urinary excretion. But, even if it were proved to be a diuretic, it would be the merest hypothesis that it eliminated syphilitic poison, and we should have to explain how if came to act so differently to other unquestionable diuretics. To my own mind, the essential agency of iodide of potassium, as of mercury, lies in its nullifying inflammatory nisus; but it has a more inmediate relation than the latter to syphilitic and rheumatic inflammations. It appears to me a fact full of significance, that a man who has contracted syphilis, and been apparentiy cured of it, may remain well for a considerable time, till something breaks down his health, and then the symptoms reappear. Does not this show that, while the vitality of the tissues is vigorous, they can tolerate the presence of the poison without injury; but, as soon as they are enfeebled, the rnorbid action recommences? The curious facts observed in the treatment of syphilis by repeated inoculation also point out that the expulsion of the poison is not the sole and only means of obtaining immunity from its injurious influence.

With regard to ordinary skin-diseases, I think the belief has been and is very general, that they depend on some materies morb $i$ acting as a cause of irritation, which requires to be either neutralised or eliminated. But, taking the eruptions which show the greatest tendency to effusion of fluid, such as pemphigus and eczema, do we not certainly find that the best treatment is one which, by toning the vessels of the part affected, arrests the discharge; and that this, which we can often do by arsenic, so far from disordering, improves the general health. What is true of these eruptions is also true in a less degree of most others, always provided that the inflammatory action is not of a sthenic kind, for in that case we 